\title{
Mineral oil certified reference materials for the determination of polychlorinated biphenyls from the National Metrology Institute of Japan (NMIJ)
}

\author{
Masahiko Numata Yoshie Aoyagi • Mayumi Matsuo • \\ Keiichiro Ishikawa $\cdot$ Nobuyasu Hanari • \\ Satoko Otsuka $\cdot$ Yoko Tsuda $\cdot$ Takashi Yarita
}

Received: 30 December 2007 / Revised: 14 February 2008/Accepted: 20 February 2008 / Published online: 16 April 2008

(C) Springer-Verlag 2008

\begin{abstract}
Four mineral oil certified reference materials (CRMs), NMIJ CRM 7902-a, CRM 7903-a, CRM 7904-a, and CRM 7905-a, have been issued by the National Metrology Institute of Japan, which is part of the National Institute of Advanced Industrial Science and Technology (NMIJ/AIST), for the determination of polychlorinated biphenyls (PCBs). The raw materials for the CRMs were an insulation oil (CRM 7902-a and CRM 7903-a) and a fuel oil (CRM7904-a and CRM 7905-a). A solution of PCB3, $\mathrm{PCB} 8$, and technical PCB products, comprising four types of Kaneclor, was added to the oil matrices. The total PCB concentrations in the PCB-fortified oils (CRM 7902-a and CRM 7904-a) are approximately $6 \mathrm{mg} \mathrm{kg}^{-1}$. In addition, the mineral oils which were not fortified with PCBs were also distributed as CRMs (CRM 7903-a and CRM 7905-a). Characterization of these CRMs was conducted by the NMIJ/AIST, where the mineral oils and the PCB solution were analyzed using multiple analytical methods such as dimethylsulfoxide extraction, normal-phase liquid chromatography, gel permeation chromatography, reversed-phase liquid chromatography, and chromatography using sulfoxide-bonded silica; and/or various capillary columns for gas chromatography, and two ionization modes for mass
\end{abstract}

Electronic supplementary material The online version of this article (doi:10.1007/s00216-008-2010-3) contains supplementary material, which is available to authorized users.

M. Numata $(\bowtie) \cdot$ Y. Aoyagi $\cdot$ M. Matsuo $\cdot$ K. Ishikawa

N. Hanari $\cdot$ S. Otsuka $\cdot$ Y. Tsuda $\cdot$ T. Yarita

Organic Standards Section 2,

National Metrology Institute of Japan (NMIJ) National Institute of Advanced Industrial Science and Technology (AIST),

1-1-1, Umezono, Tsukuba,

Ibaraki, 305-8563, Japan

e-mail: mas-numata@aist.go.jp spectrometry. The target compounds in the mineral oils and those in the PCB solution were determined by one of the primary methods of measurement, isotope dilutionmass spectrometry (ID-MS). Certified values have been provided for 11 PCB congeners (PCB3, 8, 28, 52, 101, 118, $138,153,180,194$, and 206) in the CRMs. These CRMs have information values for PCB homologue concentrations determined by using a Japanese official method for determination of PCBs in wastes and densities determined with an oscillational density meter. Because oil samples having arbitrary PCB concentrations between respective property values of the PCB-fortified and nonfortified CRMs can be prepared by gravimetric mixing of the CRM pairs, these CRMs can be used for validation of PCB analyses using various instruments which have different sensitivities.

Keywords Quality assurance/quality control .

Reference materials · Mineral oil · Insulation oil · Fuel oil · Polychlorinated biphenyls

\section{Introduction}

Polychlorinated biphenyls (PCBs) were once widely used as dielectric fluids, heat-transfer fluids, and hydraulic fluids, and for other applications [1]. However, some PCB congeners have dioxin-like toxicity, and more toxic products such as polychlorinated dibenzofurans are easily generated from PCB by heating. In Japan and Taiwan, Yusho disease was caused by PCB-contaminated rice bran oil and there are approximately 2,000 officially acknowledged victims in Japan [2]. After banning the production and importation of PCB products, products containing PCB and $\mathrm{PCB}$ wastes, including more than four million trans- 
formers and capacitors, have been registered and stored. Furthermore, low-level PCB contaminations were found in millions of "PCB-free" transformers in Japan. The Law Concerning Special Measures for Promotion of Proper Treatment of PCB Waste enforced in 2005 provides treatment of all PCBs stored in Japan by 2016 [3]. Although accurate determination of PCB concentration is essential to estimate their risk and to manage PCB waste treatment properly, accurate analysis is sometimes difficult because residual matrices such as hydrocarbons sometimes interfere with PCB measurements and because of overlap between PCB congeners themselves. In addition, the criterion of PCB concentration in waste (oil) in Japan $\left(0.5 \mathrm{mg} \mathrm{kg}^{-1}\right)$ is strict compared with those in most of countries $\left(2-50 \mathrm{mg} \mathrm{kg}^{-1}\right)$.

For reliability of the analytical results of trace pollutants, quality assurance of analyses using certified reference materials (CRMs) is essential. Not only standard solutions for calibrations of analytical instruments but also matrix-type CRMs are required to validate analytical results of actual samples, because matrix effects may result in bias of the analytical results. The National Metrology Institute of Japan, which is part of the National Institute of Advanced Industrial Science and Technology (NMIJ/AIST), has prepared matrix CRMs for environmental analysis $[4,5]$. Certifications of these matrix CRMs were mainly conducted by the NMIJ/ AIST only, using a primary method of measurement [6], isotope dilution-mass spectrometry (ID-MS), to establish traceability of their property values to the SI system. Although the potential of ID-MS is high, inadequate analytical conditions such as low recovery, contamination, and insufficient resolution in chromatography separation cause biased results. Therefore, the certified values are decided from the analytical results obtained by two or more well-validated and/or optimized analytical methods.

Recently, four mineral oil reference materials for the determination of PCBs have been prepared and certified by the NMIJ/AIST. Development and supply of such national CRMs are important to allow compliance with regulations set by Japanese laws and domestic pollution patterns, and to eliminate complex customs procedures for hazardous chemicals in foreign CRMs.

Because the most abundant PCB-containing waste is insulation oil (transformer oil), we have developed insulation oil CRMs of which the main composition is paraffin oil. However, sometimes compositions of waste oils are more complex. Therefore we have also prepared fuel oil CRMs which contains naphthene, aromatics, asphaltene, and others. The mineral oil CRMs with two PCB concentration levels have been prepared because PCBs are determined with various instruments having different sensitivities. Users can adjust PCB concentration arbitrarily by gravimetric mixing.
There are ten PCB homologue groups that differ from one another in the number of chlorine atoms, and theoretically 209 PCB congeners exist. More than 100 congeners having different toxicities and chemical or physical properties exist in the environment and $\mathrm{PCB}$ formulations (technical $\mathrm{PCBs}$ ). In addition, $\mathrm{PCBs}$ in environmental or industrial wastes are regulated in accordance with total PCB concentration set by Japanese environment laws. Thus, we provided certified values for at least one major congener from each homologue group except decachlorinated biphenyl, and information values for ten homologue groups. The selected congeners are also used for gas chromatography/mass spectrometry (GC/MS) calibration standards prescribed by a Japanese official method for the determination of PCBs in waste [7]. Determinations of the certified values of these target compounds were performed by isotope dilution-gas chromatography/mass spectrometry (ID-GC/MS), and more than two types of analytical techniques were applied in each step. In this paper, the preparation and the certification of four mineral oil CRMs for the determination of polychlorinated biphenyls (NMIJ CRM 7902-a, polychlorinated biphenyls in insulation oil (high concentrations); CRM 7903-a, polychlorinated biphenyls in insulation oil (low concentrations); CRM 7304-a, polychlorinated biphenyls in fuel oil; CRM 7905-a, polychlorinated biphenyls in fuel oil (PCB blank)) are described.

\section{Experimental}

\section{Chemicals}

Surrogate solutions and syringe spike solutions were prepared from solutions of each isotope-labeled compound $\left({ }^{13} \mathrm{C}_{12}\right.$-labeled PCB3, 8, 15, 28, 52, 101, 118, 138, 153, 170, 180, 194, 206, and 209: $50 \mu \mathrm{g} \mathrm{mL}^{-1}$, Wellington Laboratories, Guelph, Canada). Calibration solutions for GC/MS analysis were prepared from neat reagents (PCB8, 52, 101, 118, 138, and 180, AccuStandard, New Haven, CT, USA; PCB3, 206, and 209, Cambridge Isotope Laboratories, Andover, MA, USA) and the isotope-labeled reagent solutions described above. To prepare a PCB solution for spiking of PCBs to the oil materials (PCB "root" solution), PCB3 and PCB8 reagents (Chem Service, West Chester, PA, USA) and technical PCBs comprising four types of Kaneclor (KC300, KC400, KC500, and KC600; GL Sciences, Tokyo, Japan) were used. The PCB congener numbers used in this article are IUPAC numbers [8].

PCB and pesticide analysis grade solvents (hexane, acetone, and acetonitrile; Kanto Chemical, Tokyo, Japan) and reagent grade solvents (nonane and dimethylsulfoxide; Kanto Chemical) were used for the analytical procedures 
and preparation of solutions. Other reagents were obtained from Wako Pure Chemical Industries or Kanto Chemical. The water used for this work was purified using a Milli-Q Element system (Nippon Millipore Kogyo, Tokyo, Japan). High-purity methane gas $(>99.999 \%$, Takachiho Chemical Industrial, Tokyo, Japan) was used as a reaction gas for negative chemical ionization (NCI) mode.

Preparation of candidate reference materials

Commercial insulation oil (provided in a Japanese Industrial Standard, JIS C2320 class 1-2) was collected from a transformer $(300 \mathrm{KVA}, 6.6 \mathrm{kV} / 210 \mathrm{~V})$ that had been used for 26 years. The collection and transportation were done by a collaborator, General Environmental Technos (Tokyo, Japan). This insulation oil or a commercial fuel oil (provided in JIS K2205 class 1-2) was filtered through a glass fiber filtration paper, GC50 (diameter $47 \mathrm{~mm}$, thickness $0.19 \mathrm{~mm}$; Advantec Toyo Kaisha, Tokyo, Japan), and then 10-mL aliquots of the oil were sealed in 20-mL-volume amber ampoules together with argon gas: these are the "base oil" CRMs, CRM 7903-a and CRM 7905-a. Ampouling was carried out using an automatic filling and sealing machine, Model IMC-1407 (Imoto Machinery, Kyoto, Japan).

To prepare the PCB-fortified oil CRMs, CRM 7902-a and CRM 7904-a, the PCB3, PCB8 reagent, and technical PCBs, comprising Kaneclor, were dissolved in nonane, then $0.3 \mathrm{~g}$ of the PCB root solution was added gravimetrically to $1.5 \mathrm{~kg}$ of the above filtered oils (final concentration, CRM 7902-a: PCB3, $0.501 \mathrm{mg} \mathrm{kg}^{-1}$; PCB8, $0.504 \mathrm{mg} \mathrm{kg}$; $\mathrm{KC} 300,1.27 \mathrm{mg} \mathrm{kg}^{-1}$; KC400, $1.29 \mathrm{mg} \mathrm{kg}^{-1}$; KC500, $1.29 \mathrm{mg} \mathrm{kg}^{-1}$; and KC600, $1.28 \mathrm{mg} \mathrm{kg}^{-1}$ oil; CRM 7904-a: PCB3, $0.501 \mathrm{mg} \mathrm{kg}^{-1}$; $\mathrm{PCB} 8,0.503 \mathrm{mg} \mathrm{kg}^{-1}$; KC300, $1.25 \mathrm{mg} \mathrm{kg}^{-1} ; \mathrm{KC} 400,1.27 \mathrm{mg} \mathrm{kg}^{-1} ; \mathrm{KC} 500$, $1.27 \mathrm{mg} \mathrm{kg}^{-1}$; and $\mathrm{KC} 600,1.27 \mathrm{mg} \mathrm{kg}^{-1}$ ). The PCBfortified oils were homogenized in a 2-L-volume amber glass bottle with a rocking mixer for $6 \mathrm{~h}$. Completion of the homogenization was checked by analyses of PCB concentrations in the mixture of PCBs and the insulation oil taken from six different positions in the bottle. Because the fuel oil was less viscous than the insulation oil, the preliminary homogeneity test for the fuel oil was omitted. The mixture $(2.5 \mathrm{~mL})$ was sealed in 5-mL-volume amber ampoules as described above.

Assay for the purity of PCB reagents

The purities of the PCB reagents were evaluated by the relative peak area percentages of main and minor components using a gas chromatography/flame ionization detector (GC/FID) system, GC-2010 (Shimadzu, Kyoto, Japan). The impurities were identified with a gas chromatography quadrupole mass spectrometer (GC/QMS) system, Agilent
6890GC/5973N MSD (Agilent Technologies, Palo Alto, CA, USA). The water content in the reagents was evaluated with a Karl Fisher coulometric titrator, MKC-510 (Kyoto Electronics Manufacturing, Kyoto, Japan).

Preparation of surrogate solutions and GC/MS calibration solutions

In order to prepare GC/MS calibration solutions with a well-defined concentration, an assay for the purity of the natural isotope abundance ("native") PCB reagents used for preparing the standard solutions was carried out. The amounts of impurities such as PCB congeners and polychlorinated terphenyls in the $\mathrm{PCB}$ reagents were estimated by GC/FID, the impurities were identified by GC/QMS, and response factors of the impurities on FID (area of peak on the FID chromatogram/mol of impurity/number of carbon atom in the impurity molecule) were corrected. Although the water contents in the reagents were not detected in most cases, the level of detection limit (LOD) was used to estimate uncertainty of the purity.

For the determination of some PCB congeners, standard solutions issued by NMIJ, CRM 4206-a1 (CB28, 10.04士 $0.17 \mathrm{mg} \mathrm{kg}^{-1}$ in 2,4,4-trimethylpentane), CRM 4207-a1 (CB153, 10.13 $\pm 0.17 \mathrm{mg} \mathrm{kg}^{-1}$ in 2,4,4-trimethylpentane), and CRM 4209-a1 (CB194, 9.99 $\pm 0.16 \mathrm{mg} \mathrm{kg}^{-1}$ in 2,4,4trimethylpentane) were used for the preparation of the calibration solution instead of the respective neat reagents.

The calibration solutions and surrogate solutions were prepared by gravimetric mixing of the neat reagents, the standard solutions, and nonane. To keep relative composition of isotope-labeled compounds constant between calibration solution and surrogate solution, the stock solution of isotopelabeled compound mixture was mixed with the stock solution of the native compounds or solvent to prepare the calibration solution or the surrogate solution, respectively, at the final step of the preparation. The final concentration of each component in the sample solution for the PCB-fortified oils (cleaned extract for GC/MS measurement: mixture of the surrogate and the native target compounds in the sample) and the GC/MS calibration solution were adjusted almost the same. For the base oil CRMs, the surrogate solution and the calibration solution were diluted to $1: 100$.

Analytical methods for characterization

The analytical procedures were validated to optimize sensitivity and selectivity. Removal of the sample matrices is especially important for the cleanup step to minimize interference with GC/MS measurements. In addition to the official method (dimethylsulfoxide (DMSO) extraction [7, 9]), chromatographic techniques (normal-phase liquid chromatography [10, 11], gel permeation chromatography [12], 
reversed-phase liquid chromatography [13]) have been evaluated, and were used for the characterizations. A new stationary phase for liquid chromatography, sulfoxidebonded silica [14], was also used for the analysis. Separation efficiencies of PCBs from the mineral oils by the chromatographic techniques were mainly estimated from their elution profiles. To determine elution profiles of the mineral oils in the chromatography columns, the collected fractions were evaporated to dryness with a nitrogen gas stream, and the oil recoveries were calculated from the mass of the evaporation residue and the mass of the loaded oil. For the determination of PCB elution profiles, the volume of each eluted fraction was reduced to $0.2-1.0 \mathrm{~mL}$ under a nitrogen gas stream, and $\mathrm{PCB}$ concentrations in the fractions were measured by GC/MS.

For the characterization of CRM 7903-a and CRM 7905a, the samples were analyzed by using the above cleanup procedures. On the other hand, certified values of CRM 7902-a or CRM 7904-a were calculated from PCB concentrations in a PCB root solution determined by combinations of four kinds of GC column and GC/MS ionization modes (electron ionization (EI) and NCI [15]), PCB concentrations in CRM 7903-a or CRM 7905-a (oil matrix), and mass ratio between the PCB spike solution and oil matrix for the preparation.

Analytical schemes for the determination of the PCBs in the CRMs are summarized in Fig. 1.

\section{DMSO extraction procedures}

The analytical procedures basically followed a Japanese official method for the determination of PCBs in wastes [7].
After the addition of a surrogate solution $(0.2 \mathrm{~mL})$ to the oil sample $(0.2 \mathrm{~mL})$, the mixture was moved to a separatory funnel using $10 \mathrm{~mL}$ hexane. The PCB congeners in the hexane layer were extracted with $25 \mathrm{~mL}$ DMSO (beforehand saturated with hexane) four times. The DMSO layers were combined and washed with $100 \mathrm{~mL}$ hexane. Then, $100 \mathrm{~mL}$ water was added to the DMSO layer, and the mixture was extracted with $65 \mathrm{~mL}$ hexane three times.

In the case of the insulation oil samples, the hexane layer was then washed with $10 \mathrm{~mL}$ of $2 \mathrm{M} \mathrm{KOH}$ aqueous solution. After washing with $25 \mathrm{~mL}$ water twice, the hexane layer was dried with $5 \mathrm{~g}$ anhydrous sodium sulfate, and concentrated to ca. $100 \mathrm{~mL}$ with a rotary evaporator. After shaking with $25 \mathrm{~mL}$ sulfuric acid (98\%) four times and with $25 \mathrm{~mL}$ water twice, the hexane layer was dried with $5 \mathrm{~g}$ anhydrous sodium sulfate, and concentrated to $1 \mathrm{~mL}$ with a rotary evaporator and under a nitrogen gas stream. The solution was then passed through a glass column (10-mm i.d.) containing $3 \mathrm{~g}$ silica gel (particle size 70-150 $\mu \mathrm{m}$; Wakogel C-200, Wako Chemical, after washing with methanol, dried at $130{ }^{\circ} \mathrm{C}$ for $18 \mathrm{~h}$ ) capped with $1.5 \mathrm{~g}$ anhydrous sodium sulfate to remove polar constituents. After conditioning with $50 \mathrm{~mL}$ hexane, the sample solution was loaded, and PCB congeners were recovered with $130 \mathrm{~mL}$ hexane.

In the case of the fuel oil samples, the hexane layer recovered by the back-extraction from the DMSO extract was treated with a multilayered column to reduce contaminants more effectively. The hexane layer was dried with $5 \mathrm{~g}$ anhydrous sodium sulfate, and concentrated to $2 \mathrm{~mL}$ with a rotary evaporator and under a nitrogen gas stream. The solution was then passed through a multilayer column (25-mm-i.d. polypropylene cartridge containing $3 \mathrm{~g}$ of $10 \%$
Fig. 1 Analytical scheme for the characterization of the NMIJ mineral oil CRMs. rp-HPLC, reversed-phase HPLC; np-HPLC, normal-phase HPLC

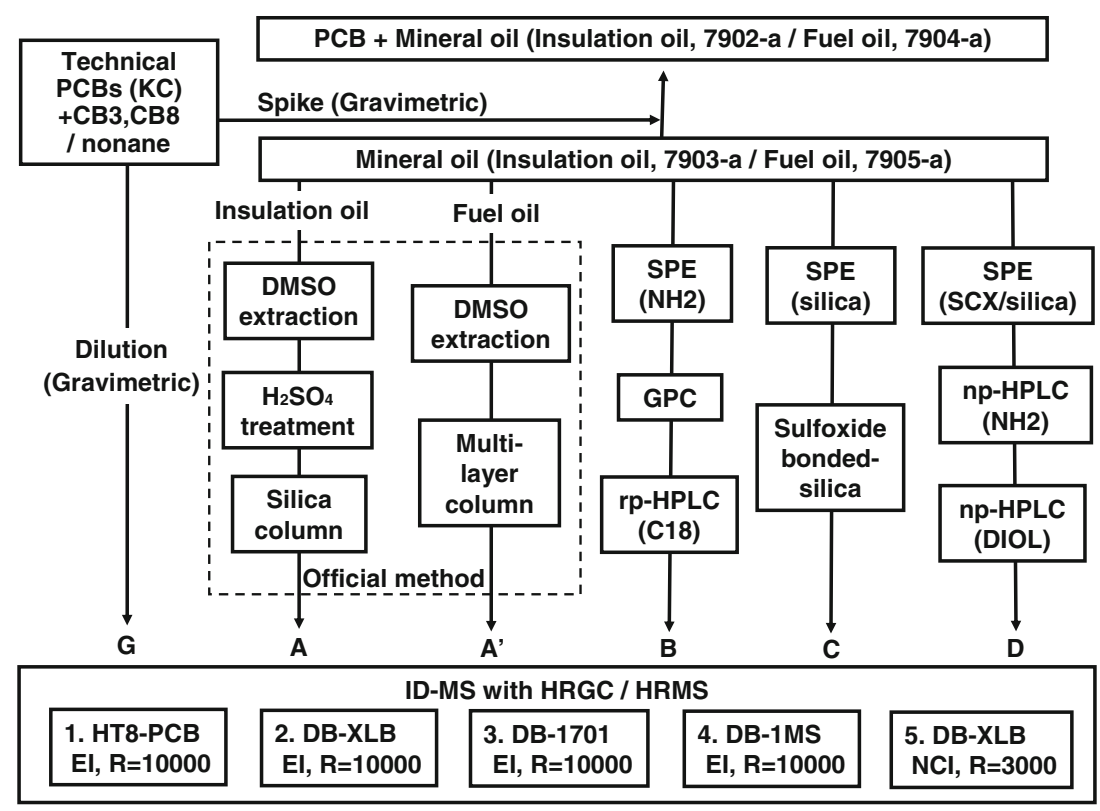


$\mathrm{AgNO}_{3} /$ silica gel, $4.5 \mathrm{~g}$ of $22 \% \mathrm{H}_{2} \mathrm{SO}_{4} /$ silica gel, $4.5 \mathrm{~g}$ of $44 \% \mathrm{H}_{2} \mathrm{SO}_{4} /$ silica gel, $3 \mathrm{~g}$ of $2 \% \mathrm{KOH} /$ silica gel; GL Sciences, Tokyo, Japan). After conditioning with $500 \mathrm{~mL}$ hexane/dichloromethane mixture $(24: 1 \mathrm{v} / \mathrm{v})$, the sample solution was loaded, and PCB congeners were recovered with $300 \mathrm{~mL}$ of the solvent mixture.

The fraction containing PCBs was concentrated to $0.2 \mathrm{~mL}$ with a rotary evaporator and under a nitrogen gas stream, and the fraction was analyzed by GC/MS.

Gel permeation chromatography (GPC) and reversed-phase liquid chromatography cleanup procedures

The oil sample $(0.2 \mathrm{~mL})$ was placed in a test tube, and the surrogate solution $(0.2 \mathrm{~mL})$ was added. The solution was passed through a solid-phase extraction cartridge $(1 \mathrm{~g}$ aminopropylsilica-packed 3-mL cartridge, ISOLUTE-NH2; International Sorbent Technology, Hengoed, UK, conditioned with $10 \mathrm{~mL}$ hexane). PCBs were then recovered with $11.5 \mathrm{~mL}$ hexane. The GPC cleanup was performed by the method of Matsubara et al. [12] with some modifications. The SPE-treated solution was concentrated to $0.4 \mathrm{~mL}$ under a nitrogen gas stream and the solvent was replaced with acetone. Then, $0.2 \mathrm{~mL}$ of the solution was injected into a GPC system (CLASS-VP, Shimadzu, Kyoto, Japan; equipped with a styrene-divinylbenzene column, $300 \mathrm{~mm} \times 20$-mm i.d., CLNpack PAE-200AC and a guard column, CNLpack PAEGAC; Shodex, Tokyo, Japan, column temperature $30^{\circ} \mathrm{C}$ ). The PCBs were eluted with acetone at the flow rate of $4.0 \mathrm{~mL} \mathrm{~min}{ }^{-1}$. The fraction containing PCBs (14.3$18.9 \mathrm{~min}$ ) was concentrated to $0.4 \mathrm{~mL}$ with a rotary evaporator and under a nitrogen gas stream, and the solvent was replaced with acetonitrile. Then, $0.35 \mathrm{~mL}$ of the solution was injected into a reversed-phase liquid chromatography system (Gulliver System, JASCO, Tokyo, Japan; equipped with a octadecylsilane-silica gel (Wakosil 40C18, average particle diameter $40 \mu \mathrm{m}$; spherical particle; Wako Pure Chemical, Tokyo, Japan) packed stainless steel column (250 $\mathrm{mm} \times 7.6-\mathrm{mm}$ i.d.). The PCBs were eluted with acetonitrile at the flow rate of $1.0 \mathrm{~mL} \mathrm{~min}$. The fraction containing PCBs $(8-50 \mathrm{~min})$ was evaporated with a rotary evaporator to ca. $2 \mathrm{~mL}$. After addition of $1 \mathrm{~mL}$ acetone and $0.2 \mathrm{~mL}$ nonane, the fraction was concentrated under a nitrogen gas stream to $0.2 \mathrm{~mL}$, and the $\mathrm{PCB} /$ nonane solution was analyzed by GC/MS.

\section{Sulfoxide-bonded silica cleanup procedures}

In contrast to a previous report [14], a type of spherical aminopropylsilica (Flash-NH2; average particle diameter $50 \mu \mathrm{m}$; surface coverage $3.1 \mu \mathrm{mol} \mathrm{m} \mathrm{m}^{-2}$; carbon loading $6.0 \%$ ) was used as base material to prepare a sulfoxidebonded silica stationary phase instead of the irregular shaped silica. The sulfoxide residues were bonded by the method described in the report. Compared with the irregular shaped stationary phase, the separation efficiencies of the spherical stationary phase were improved (resolution factors between PCB congeners and eicosane: PCB3, 4.52 0.02; PCB101, 4.18 $\pm 0.01 ; \mathrm{PCB} 209,2.84 \pm 0.03 ; n=3$, mean \pm SD under the same chromatographic conditions used in the ref. [14]).

The oil sample $(0.2 \mathrm{~mL})$ was placed in a test tube, and the surrogate solution $(0.2 \mathrm{~mL})$ was added. The solution was passed through a solid-phase extraction cartridge (500 mg silica-packed 3-mL cartridge, ISOLUTE-SILICA; International Sorbent Technology, Hengoed, UK, conditioned with $10 \mathrm{~mL}$ hexane). Then PCBs were then recovered with $11.5 \mathrm{~mL}$ hexane. The obtained fraction was concentrated to $0.4 \mathrm{~mL}$ under a nitrogen gas stream and then $0.1 \mathrm{~mL}$ of the solution was injected into a normal-phase liquid chromatography system (LC-10, Shimadzu, Kyoto, Japan; equipped with a stainless steel column $(150 \mathrm{~mm} \times 7.6-\mathrm{mm}$ i.d.) packed with the sulfoxide-bonded silica. After the packing and PCB recovery from the samples, the stationary phase was washed with $6.0 \mathrm{~mL}$ acetone to remove possible polar contaminants, and was then washed with more than $40 \mathrm{~mL}$ hexane to remove acetone. The PCBs were eluted with hexane at the flow rate of $0.6 \mathrm{~mL} \mathrm{~min}$. The fraction containing PCBs (15-35 min) was concentrated to $0.2 \mathrm{~mL}$ with a rotary evaporator and nitrogen gas stream, and the fraction was analyzed by GC/MS.

Normal-phase liquid chromatography cleanup procedures

The procedure used was modified from the method reported by Tanaka et al. [11]. The oil sample $(0.2 \mathrm{~mL})$ was placed in a test tube, and the surrogate solution $(0.2 \mathrm{~mL})$ was added. The solution was passed through a solid-phase extraction cartridge ( $1 \mathrm{~g}$ benzene sulfonic acid-bonded silica/silicapacked 3-mL cartridge, Bond Elute PCB; Varian, Palo Alto, CA, USA; conditioned with $10 \mathrm{~mL}$ hexane). PCBs were then recovered with $11.5 \mathrm{~mL}$ hexane. The obtained fraction was concentrated to $0.4 \mathrm{~mL}$ under a nitrogen gas stream and then $0.2 \mathrm{~mL}$ of the solution was injected into a normal-phase liquid chromatography system (LC-10, Shimadzu; equipped with an aminopropylsilica column, $100 \mathrm{~mm} \times 7.6-\mathrm{mm}$ i.d.; Inertsil NH2, GL Sciences). The PCBs were eluted with hexane at the flow rate of $2.0 \mathrm{~mL} \mathrm{~min}{ }^{-1}$. The fraction containing PCBs (2.5-8.5 min) was concentrated to $0.4 \mathrm{~mL}$ with a rotary evaporator and nitrogen gas stream, and then $0.35 \mathrm{~mL}$ of the solution was injected into another normalphase liquid chromatography system (LC-10, Shimadzu; equipped with a dihydroxypropylsilica column, $250 \mathrm{~mm} \times$ 7.6-mm i.d.; Inertsil DIOL, GL Sciences). The PCBs were eluted with hexane at the flow rate of $2.0 \mathrm{~mL} \mathrm{~min}{ }^{-1}$. The fraction containing PCBs (5.5-11.5 min) was concentrated 
to $0.2 \mathrm{~mL}$ with a rotary evaporator and nitrogen gas stream, and the fraction was analyzed by GC/MS.

\section{Determination of PCBs by GC/HRMS}

Analyses of PCBs were performed using a gas chromatography-high-resolution mass spectrometry (GC/HRMS) system, AutoSpec (Micromass, Manchester, UK) or JMS700D (JEOL, Tokyo, Japan). After $50 \mu \mathrm{L}$ of the syringe spike solution $\left(\left[{ }^{13} \mathrm{C}_{12}\right] \mathrm{PCB} 15\right.$ for evaluation of recovery yields of monochlorinated to pentachlorinated biphenyl surrogates, and $\left[{ }^{13} \mathrm{C}_{12}\right] \mathrm{PCB} 170$ for hexachlorinated to decachlorinated biphenyl surrogates) was added to the cleaned fraction obtained by each method, the solution volume was reduced to $0.2 \mathrm{~mL}$ by means of a rotary evaporator and under a nitrogen gas stream. To determine PCB concentration in the root PCB solution, the solution was diluted gravimetrically with nonane to form a $\mathrm{PCB}$ solution whose $\mathrm{PCB}$ concentration was nearly the same as that of the PCB-fortified CRMs; the surrogate solution was then added to a portion of the diluted solution.

A portion of the solution $(1.0 \mu \mathrm{L})$ was injected in the splitless mode. The analytes were separated using a gas chromatograph (Agilent 6890, Agilent Technologies, Palo Alto, CA, USA) equipped with a capillary column. The columns used for the separation were as follows: HT8-PCB $(60 \mathrm{~m} \times 0.25-\mathrm{mm}$ i.d., film thickness not reported; Kanto Chemical), DB-XLB $(60 \mathrm{~m} \times 0.25$-mm i.d., film thickness $0.25 \mu \mathrm{m}$; Agilent Technologies), DB-1701 (30 m×0.25$\mathrm{mm}$ i.d., film thickness $0.25 \mu \mathrm{m}$; Agilent Technologies), and DB-1MS ( $30 \mathrm{~m} \times 0.25$-mm i.d., film thickness $0.25 \mu \mathrm{m}$; Agilent Technologies). PCB congeners were detected in EI or NCI mode. For the determination of total PCB homologue groups, two injections were necessary to cover all $\mathrm{PCB}$ congeners (one measurement for monochlorinated, trichlorinated, tetrachlorinated, heptachlorinated, and octachlorinated biphenyls; another one for other homologue groups). The GC/MS measurement conditions are summarized in Electronic supplementary material Tables S1 and S2.

One point calibration was applied, because the linearity was good enough in the range of the solutions tested prior to the sample analyses. The concentrations of target compounds were calculated as described in the Electronic supplementary material. The uncertainties of the property values were calculated according to the "Guide to the expression of uncertainty in measurement" (GUM) [16].

\section{Homogeneity study}

The between-ampoule homogeneity of the PCB-fortified mineral oil CRMs (CRM 7902-a and CRM 7904-a) was assessed by determining PCB28, PCB153, and PCB194 in each of three subsamples taken from ten ampoules randomly selected from the lot of 600 ampoules. In the cases of the base oil CRM (CRM 7903-a), analytical values of PCB52, PCB118, and PCB138 were used. The target compounds were determined by normal-phase liquid chromatography-GC/HRMS methods (CRM 7903-a, method D-2; CRM 7902-a and CRM 7904-a; a combination of silica and $\mathrm{NH} 2$ columns were used to reduce analysis time). The uncertainties associated with sample inhomogeneity were estimated by the method described in the refs. [17] and [18] (see Electronic supplementary material).

Information values for PCB homologues and density

The mass fractions of PCB homologues were the results of analysis by the Japanese official method for the determination of PCBs in waste [7]. For calculation of homologue concentrations, we assumed that response factors of $\mathrm{PCB}$ isomers in each homologue group were the same as those of the isomer used for the calibration solution. Information values for density were obtained with an oscillational density meter (see Electronic supplementary material).

\section{Results and discussion}

\section{Establishment of analytical methods}

As described above, we applied various combinations of cleanup procedures, GC separation columns, and ionization modes for the characterization to avoid possible bias associated with a certain analytical procedure. The cleanup procedures were validated to maximize recovery yields of the analytes, and to minimize procedural blank and interference with GC/MS measurements caused by residual contaminants. For this purpose, the effects of the cleanup conditions such as stationary phase, column size, mobile phase, and flow rate on the analytical results have been investigated. The established conditions for each cleanup technique were applied for the cleanup step in analyses for the certifications.

Combination of the DMSO extraction, sulfuric acid treatment, and column chromatography is used as the Japanese official method of PCB analysis in waste. To reduce background of the GC/MS chromatogram, the fuel oil was treated with the multilayered column instead of the silica column. Removal of sulfur compounds with $\mathrm{AgNO}_{3} /$ silica in the multilayered column was also effective to reduce the background [19].

The conventional technique, DMSO extraction, is well validated as the official method, but it is tedious and time consuming. In addition, the technique is highly dependent on the skill of the operators. Because the chromatographic technique is a relatively simple and reliable cleanup technique, recently proposed chromatographic methods 
were also used for the characterization as follows. Before these chromatographic treatment procedures, the sample solution was passed through SPE cartridges. Although the SPE treatment steps are not essential for accurate analysis, the treatments are necessary to remove polar contaminants which may irreversibly adsorb onto the stationary phases for subsequent procedures. For this purpose, three kinds of stationary phases, silica, aminopropylsilica, and benzene sulfonic acid-bonded silica/silica, were used. After the SPE treatments, most of the pigment was removed, and more than $90 \%$ of matrices and PCBs remained.

By GPC, compounds are eluted in the order of the reverse of their molecular sizes (i.e., largest first). Therefore GPC is popular for separating relatively low molecular weight analytes such as pesticides from the main components in biological samples, e.g., lipids [20]. Although applications are not so numerous, GPC can also be used for separation of PCBs from mineral oils [12]. Most hydrocarbons in heavy mineral oils are eluted faster than PCBs, because of the difference between their molecular sizes. In addition, the stationary phase, styrene-divinylbenzene retains aromatics in the order of the number of benzene rings. This effect enhances separation of PCBs from aliphatic hydrocarbons. Heavier PAHs are also separated from PCB, because they are retained strongly [11]. Before the characterization, we have examined the effect of mobile phases such as acetone, acetone/acetonitrile $(1: 1 \mathrm{v} / \mathrm{v})$, acetone/ethyl acetate mixture $(1: 1 \mathrm{v} / \mathrm{v})$, acetone/cyclohexane $(1: 1 \mathrm{v} / \mathrm{v})$ on the separation. Since acetone gave the best separation between mineral oils and PCBs, acetone was used for the characterization. The elution profiles of the insulation oil, fuel oil, and some PCB homologue groups are shown in Electronic supplementary material Fig. S1. To remove remaining contaminants from $\mathrm{PCBs}$, the fraction containing PCB recovered from the GPC was treated with another chromatography run in a different mode. Preparative reversed-phase liquid chromatography has not been widely used to separate PCBs from mineral oils except in a few examples [13]. From a preliminary test for separation of PCBs from the insulation oil using the reversed-phase HPLC process, the separation efficiency was not so high (approximately $25 \%$ of hydrocarbons in typical insulation oils coeluted with PCBs under the chromatographic conditions described in the Experimental section: unpublished data). Because less polar compounds are retained more strongly, PCBs elute faster than remaining hydrocarbons (Electronic supplementary material Fig. S2A). However, the additional cleanup effect was limited for the fuel oil samples (Electronic supplementary material Fig. S2B).

Although the sulfoxide-bonded silica cleanup [14] is a kind of normal-phase liquid chromatography, specificity of the stationary phase for PCBs is much higher than other polar stationary phases which are used for cleanup of PCBs.

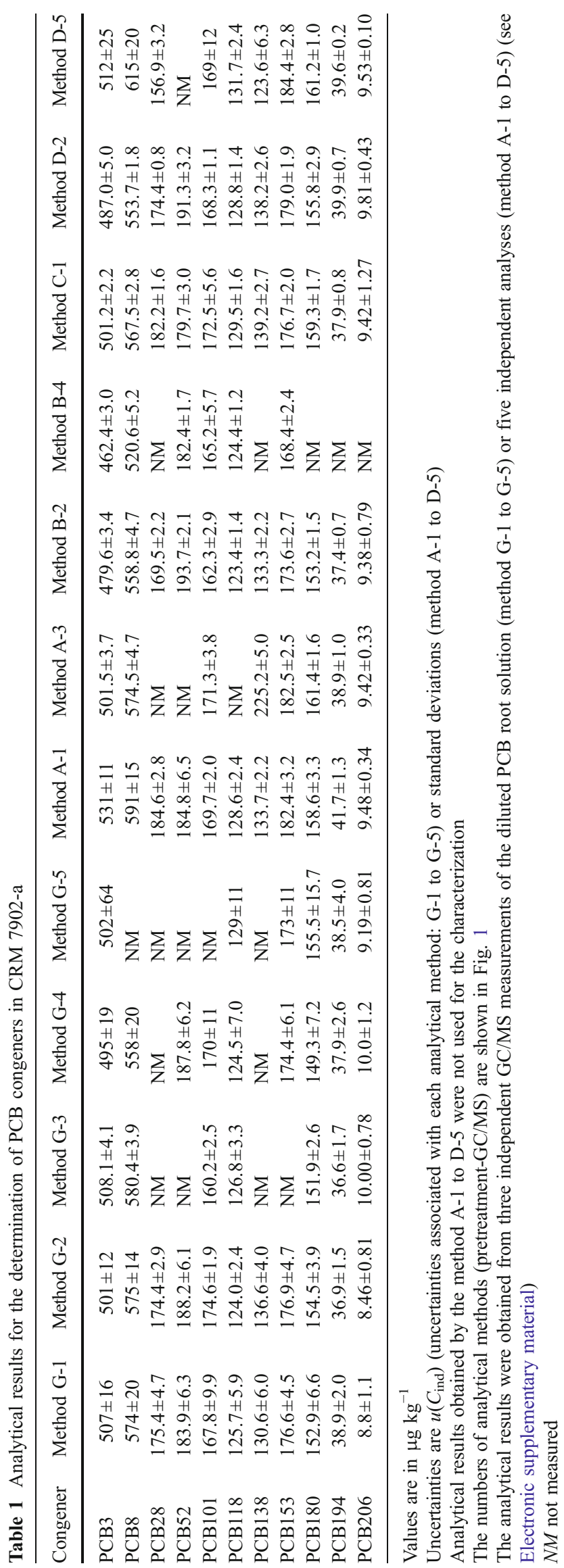


Table 2 Analytical results for the determination of PCB congeners in CRM 7903-a

\begin{tabular}{lllllcr}
\hline Congener & Method A-1 & Method A-3 & Method B-2 & Method B-4 & Method C-1 & Method D-2 \\
\hline PCB3 & $<0.224$ & NM & $<0.576$ & NM & $<0.482$ & $<0.831$ \\
PCB8 & $<0.188$ & NM & $<2.17$ & NM & $<0.855$ & $<0.865$ \\
PCB28 & $<0.204$ & NM & $<0.193$ & NM & $<0.439$ & NM \\
PCB52 & $0.345 \pm 0.024$ & NM & $0.304 \pm 0.080$ & NM & 0.181 \\
PCB101 & $0.329 \pm 0.063$ & $0.220 \pm 0.061$ & $0.530 \pm 0.073$ & $0.434 \pm 0.074$ & $0.42 \pm 0.12$ & $0.420 \pm 0.086$ \\
PCB118 & $0.378 \pm 0.129$ & NM & NM & $0.424 \pm 0.051$ & $0.395 \pm 0.091$ & $0.46 \pm 0.10$ \\
PCB138 & $0.143 \pm 0.082$ & $0.342 \pm 0.082$ & NM & NM & NM \\
PCB153 & $0.155 \pm 0.069$ & $0.158 \pm 0.074$ & $0.232 \pm 0.099$ & $0.206 \pm 0.058$ & $0.130 \pm 0.083$ \\
PCB180 & $<0.125$ & NM & $<0.217$ & NM & $<0.220$ & $0.343 \pm 0.083$ \\
PCB194 & $<0.132$ & NM & $<0.150$ & NM & $<0.169$ & $<0.183$ \\
PCB206 & $<0.092$ & NM & $<0.124$ & NM & $<0.109$ & $<0.253$ \\
\hline
\end{tabular}

Values are in $\mu \mathrm{g} \mathrm{kg}^{-1}$

Uncertainties are $u\left(C_{\text {ind }}\right)$ (uncertainties associated with each analytical method)

The numbers of analytical methods (pretreatment-GC/MS) are shown in Fig. 1

The results were obtained from five independent analyses (PCB52 to PCB153) or randomly selected three data of five independent analyses of samples taken from randomly selected ampoules (other PCB congeners) (see Electronic supplementary material)

$N M$ not measured

Therefore, only two cleanup steps are necessary for the PCB determinations. PCBs are retained more strongly on the stationary phase compared with aliphatic hydrocarbons. The elution profile from the sulfoxide-bonded silica column is shown in Electronic supplementary material Fig. S3. Heavier PAHs are also more strongly retained compared with PCBs (unpublished data). On the other hand, separation of the fuel oil matrix was worse than for the insulation oil.

Normal-phase liquid chromatography cleanup procedures are popular for PCB cleanup (e.g., see refs. [10, 11]). Because many kinds of stationary phases have been used for this purpose, we had examined several kinds of polar stationary phases such as silica, cyanopropylsilica, and alumina. Among them, the dihydropropylsilica column was the best for PCB cleanup. However, aminopropyl column cleanup was also added to reduce the background of the GC/MS chromatograms. The elution profiles of PCBs and the mineral oils with the columns are shown in Electronic supplementary material Fig. S4.

Insufficient cleanup sometimes causes delay of elution of PCBs from GC capillary columns. However, significant delay and distortion of peak shapes were not observed for the sample solution treated by the above methods. The recovery yields of the spiked ${ }^{13} \mathrm{C}$-labeled $\mathrm{PCB}$ congeners in the surrogate solutions were $50-120 \%$ in most cases after correction for HPLC injection volume and remaining volume.

Analytical results obtained by the respective methods are shown in Tables 1 and 2 (insulation oil) and Electronic supplementary material Tables S3 and S4 (fuel oil). Deviations of the results obtained by direct analyses of the PCB-fortified CRMs (method A-1 to D-5 in Table 1 and Electronic supplementary material Table S3) were larger than those of the results obtained by analyses of the PCB solution, especially for the lightly chlorinated biphenyls. To
Table 3 Certified values for PCB congeners in NMIJ CRM 7902-a and CRM 7904-a

The results are expressed as the certified concentration \pm expanded uncertainty $(k=2)$ The numbers of analytical methods (pretreatmentGC/MS) are shown in Fig. 1

\begin{tabular}{llll}
\hline \multirow{2}{*}{ Congener } & \multicolumn{2}{c}{ Certified value $\left(\mu \mathrm{g} \mathrm{kg}^{-1}\right)$} & Analytical method \\
\cline { 2 - 3 } & CRM 7902-a & CRM 7904-a & \\
\hline PCB3 & $505 \pm 21$ & $505 \pm 20$ & G-1, G-2, G-3, G-4, G-5 \\
PCB8 & $576 \pm 29$ & $575 \pm 29$ & G-1, G-2, G-3, G-4 \\
PCB28 & $174 \pm 7$ & $173 \pm 8$ & G-1, G-2 \\
PCB70 & $187 \pm 9$ & $184 \pm 10$ & G-1, G-2, G-4 \\
PCB101 & $169 \pm 13$ & $167 \pm 13$ & G-1, G-2, G-3, G-4 \\
PCB118 & $126 \pm 7$ & $124 \pm 7$ & G-1, G-2, G-3, G-4, G-5 \\
PCB138 & $134 \pm 11$ & $133 \pm 14$ & G-1, G-2 \\
PCB153 & $176 \pm 7$ & $174 \pm 7$ & G-1, G-2, G-3, G-4, G-5 \\
PCB180 & $153 \pm 8$ & $151 \pm 8$ & G-1, G-2, G-3, G-4, G-5 \\
PCB194 & $38 \pm 3$ & $37 \pm 3$ & G-1, G-2, G-3, G-4, G-5 \\
PCB206 & $9.3 \pm 1.7$ & $9.2 \pm 1.6$ & G-1, G-2, G-3, G-4, G-5 \\
& & & \\
\hline
\end{tabular}


Table 4 Certified values for PCB congeners in NMIJ CRM 7903-a and CRM 7905-a
The results are expressed as the certified concentration \pm expanded uncertainty $(k=2)$ The numbers of analytical methods (pretreatmentGC/MS) were shown in Fig. 1

\begin{tabular}{|c|c|c|c|c|}
\hline \multirow[t]{2}{*}{ Congener } & \multicolumn{2}{|l|}{ CRM 7903-a } & \multicolumn{2}{|l|}{ CRM 7905-a } \\
\hline & $\begin{array}{l}\text { Certified value } \\
\left(\mu \mathrm{kg}^{-1}\right)\end{array}$ & Analytical method & $\begin{array}{l}\text { Certified value } \\
\left(\mu \mathrm{g} \mathrm{kg}^{-1}\right)\end{array}$ & $\begin{array}{l}\text { Analytical } \\
\text { method }\end{array}$ \\
\hline PCB3 & $<0.22$ & A-1 & $<0.88$ & $\mathrm{C}-1$ \\
\hline PCB8 & $<0.19$ & A-1 & $<0.86$ & $\mathrm{C}-1$ \\
\hline PCB28 & $<0.20$ & A-1 & $<0.21$ & $\mathrm{~A}^{\prime}-1$ \\
\hline PCB70 & $0.33 \pm 0.12$ & A-1, B-2, D-2 & $<0.11$ & $\mathrm{~A}^{\prime}-1$ \\
\hline PCB101 & $0.38 \pm 0.24$ & $\mathrm{~A}-1, \mathrm{~A}-3, \mathrm{~B}-2, \mathrm{~B}-4, \mathrm{C}-1, \mathrm{D}-2$ & $<0.15$ & $A^{\prime}-1$ \\
\hline PCB118 & $0.42 \pm 0.15$ & A-1, B-2, C-1, D-2 & $<0.14$ & $A^{\prime}-1$ \\
\hline PCB138 & $0.27 \pm 0.23$ & A-1, B-2, D-2 & $<0.23$ & $\mathrm{~A}^{\prime}-1$ \\
\hline PCB153 & $0.21 \pm 0.14$ & $\mathrm{~A}-1, \mathrm{~A}-3, \mathrm{~B}-2, \mathrm{~B}-4, \mathrm{C}-1, \mathrm{D}-2$ & $<0.23$ & $\mathrm{~A}^{\prime}-1$ \\
\hline PCB 180 & $<0.12$ & A-1 & $<0.28$ & $\mathrm{~A}^{\prime}-1$ \\
\hline PCB194 & $<0.13$ & A-1 & $<0.32$ & $\mathrm{~A}^{\prime}-1$ \\
\hline РCB206 & $<0.09$ & A-1 & $<0.15$ & $\mathrm{~A}^{\prime}-1$ \\
\hline
\end{tabular}

reduce uncertainties associated with method bias, the results obtained by the direct analyses were omitted from the determination of certified values. Although the uncertainties associated with the difference of the analytical methods were larger in the case of the fuel oil, the differences were not significant for trichlorinated to nonachlorinated biphenyls. For the analyses of the base oil CRMs, sometimes obvious peaks (not noise) overlapping the peaks of the target compounds were observed in the cases of the chromatographic cleanup procedures (methods B to D). Thus, the official method, i.e., DMSO extraction, data were used to estimate LODs, namely certified values of undetectable congeners. However, small amounts of lightly chlorinated biphenyls or unknown compounds which overlapped lightly chlorinated biphenyl peaks on the GC/MS chromatogram were eluted from the multilayered column with the procedural blank test, even after conditioning with $500 \mathrm{~mL}$ of the elution solvent. This made the LOD of lightly chlorinated biphenyls in the fuel oil high (Electronic supplementary material Table S4). Therefore, data of the method C-1 (sulfoxidebonded silica cleanup) were used to determine LODs of PCB3 and PCB8 in the CRM 7905-a. By other methods, many peaks of residual contaminants were observed near the target compounds, lightly chlorinated biphenyls.
Selection of the separation column for the GC/MS measurement is especially important for PCB determination, because some PCB congeners may overlap each other on the chromatogram. At first, possible overlaps of peaks were picked up from refs. [21, 22] in which all PCB congeners are assigned. Then, elution profiles of the $\mathrm{PCB}$ mixture (PCB3, PCB8, and Kaneclor mixture) and some standard reagents such as PCB90 and PCB101 had been checked with the columns that were actually used for the characterization. From these results and data of PCB congener composition in technical PCBs [23, 24], possible overlapped $\mathrm{PCB}$ congeners were quantified. For example, PCB101 and PCB90 were coeluted from the DB-XLB column. To determine PCB101, data with the DB-1701 column were used to estimate peak area ratio of PCB101 and PCB90, and the data was used for the correction.

In addition to EI mode, NCI mode was also used for determination of PCB congeners. The NCI conditions shown in Electronic supplementary material Table S2 were optimized by using a PCB solution whose PCB concentrations were higher than those in the CRMs. Obviously, sensitivity of the NCI was higher than that of EI for heptachlorinated to decachlorinated biphenyls. However, the sensitivity was not enough to obtain accurate results for

Table 5 Properties of representative mineral oil CRMs for determination of PCBs

\begin{tabular}{lllc}
\hline CRM & Matrix & $\begin{array}{l}\text { Certified components } \\
\text { (homologues) }\end{array}$ & $\begin{array}{c}\text { Range of certified values } \\
\left(\mathrm{mg} \mathrm{kg}^{-1}\right)\end{array}$ \\
\hline $\begin{array}{l}\text { NMIJ CRM 7902-a*, } \\
\text { CRM 7904-a* }\end{array}$ & $\begin{array}{l}\text { Insulation oil (transformer oil), } \\
\text { fuel oil }\end{array}$ & 11 congeners (mono- to nona-CBs) & $0.01-0.6$ \\
BAM CRM5001* & Transformer oil & 8 congeners (tetra- to hepta-CBs) & $0.11-1.4$ \\
BCR CRM420, CRM449 & Waste oil & 5 (\#420) and 10 (\#449) congeners & $0.2-1.7(\# 420) ;$ \\
& & (tri- to hepta-CBs) & $0.8-57(\# 449)$ \\
NIST SRM1581 & Motor oil, transformer oil & Total PCB & 100
\end{tabular}

*Distributed with a blank (low concentration) oil 
monochlorinated to pentachlorinated biphenyls even in the PCB-fortified oils, except PCB3 in the nonane solution and in the CRM 7902-a (Table 1 and Electronic supplementary material Table S3, methods G-5 and D-5). In addition, the lifetime of the ionization filament was significantly shortened (typically only 3 days) under the conditions.

Homogeneity and stability test

Obtained uncertainties associated with inhomogeneity of PCB28, 153, and 194 in the PCB-fortified oils (CRM $7902-$ $\mathrm{a}$ and 7904-a) were used to estimate uncertainties of monochlorinated to trichlorinated biphenyls, tetrachlorinated to hexachlorinated biphenyls, and heptachlorinated to decachlorinated biphenyls, respectively (Electronic supplementary material Tables S5 and S6). In the case of CRM 7903-a, the uncertainties associated with inhomogeneity of PCB52, 118, and 138 were used to estimate uncertainties of monochlorinated to tetrachlorinated biphenyls, pentachlorinated biphenyls, and hexachlorinated to nonachlorinated biphenyls, respectively (Electronic supplementary material Table S7). From the results, the inhomogeneities of analytes between ampoules were not significant.

Judging from similar reference materials issued from other organizations such as NIST SRM3090 [25], the expiry date of the CRMs was assigned as 10 years after the date of certification if stored at room temperature in the dark. The "ISO guide 35" [18] states that uncertainty of CRMs is estimated from standard uncertainty due to characterization, $u$ (char); standard uncertainty due to between-ampoule inhomogeneity, $u(\mathrm{bb})$; standard uncertainty due to long-term instability, $u$ (lts); and short-term instability (instability during transportation), $u$ (sts). However, we did not include the uncertainties from instability ( $u($ lts $)$ and $u($ sts $))$ in the uncertainty of the certified values, because no systematic change in concentrations of main target compounds was detected during the investigated time period (over 6 months). The stability will be further monitored at regular intervals.

\section{Certified values}

The mass fractions of PCB congeners were determined as certified values from the results of the above analyses (Tables 1 and 2 and Electronic supplementary material Tables S3 and S4). The certified values listed in Tables 3 and 4 are the weighted means of the results obtained from the analytical methods shown in Tables 1 and 2 and Electronic supplementary material Table S3.

Kaneclor 300, 400, 500, and 600 (main homologue groups are trichlorinated, tetrachlorinated, pentachlorinated, and hexachlorinated biphenyls, respectively) correspond to Aroclor 1242, 1248, 1254, and 1260, respectively. Because the NMIJ CRMs contain them, they are applicable for validating analyses of various PCB wastes. In Japan, reductive dechlorination is one of the most popular PCB treatment methods, because it can be performed in confined space under gentle conditions [3]. After the treatments, small amount of lightly chlorinated biphenyls sometimes remain. Since abundances of lightly chlorinated biphenyls in most technical PCBs are low, the NMIJ mineral oil CRMs are applicable to validate analyses of chemically degraded PCBs, because the CRMs were fortified with lightly chlorinated biphenyls, PCB3 and PCB8.

The concentration levels of the PCBs in the NMIJ CRMs are lower than that in other mineral oil CRMs such as the BAM CRM 5001 (BAM, Berlin, Germany [26]), BCR CRM 420, BCR CRM 449 (IRMM, Geel, Belgium [27, 28]), and NIST SRM1581 (NIST, Gaithersburg, MD, USA, [29]) (Table 5). The difference reflects the regulation levels in the countries.

Information values for results by the official analytical methods

The mass fraction of PCB homologue groups were the results of analyses by the official method (Table 6). Differences between the analytical results obtained by each $\mathrm{GC} /$
Table 6 Information values for PCB homologues in NMIJ CRM 7902-a, 7903-a, 7904-a, and $7905-\mathrm{a}$

*LOD for a single isomer

**Analytical method, D-5 (Fig. 1)

\begin{tabular}{lcccc}
\hline \multirow{2}{*}{ Homologue group } & \multicolumn{3}{l}{ Information value $\left(\mu \mathrm{g} \mathrm{kg}^{-1}\right)$} & \\
\cline { 2 - 5 } & CRM 7902-a & CRM 7903-a & CRM 7904-a & CRM 7905-a \\
\hline Mono-CBs & $512 \pm 33$ & $(<0.22)^{*}$ & $525 \pm 73$ & $(<0.88)^{*}$ \\
Di-CBs & $635 \pm 21$ & $0.33 \pm 0.81$ & $642 \pm 11$ & $(<0.86)^{*}$ \\
Tri-CBs & $883 \pm 146$ & $0.38 \pm 0.60$ & $871 \pm 33$ & $(<0.21)^{*}$ \\
Tetra-CBs & $1,451 \pm 421$ & $1.13 \pm 0.59$ & $1,475 \pm 195$ & $(<0.11)^{*}$ \\
Penta-CBs & $998 \pm 150$ & $2.80 \pm 0.42$ & $1047 \pm 53$ & $(<0.15)^{*}$ \\
Hexa-CBs & $961 \pm 115$ & $1.24 \pm 0.41$ & $986 \pm 22$ & $(<0.23)^{*}$ \\
Hepta-CBs & $739 \pm 89$ & $0.21 \pm 0.05$ & $732 \pm 83$ & $(<0.28)^{*}$ \\
Octa-CBs & $230 \pm 49$ & $(<0.13)^{*}$ & $229 \pm 23$ & $(<0.32)^{*}$ \\
Nona-CBs & $13.1 \pm 1.8$ & $(<0.09)^{*}$ & $12.3 \pm 0.9$ & $(<0.15)^{*}$ \\
Deca-CB & $0.14^{* *}$ & $(<0.06)^{* *}$ & $0.14 * *$ & $(<0.05)^{* *}$ \\
\hline
\end{tabular}


MS method were much larger than those of PCB congeners obtained by the isotope-dilution technique (Electronic supplementary material Tables S8-S10). Response factors of isomers in the same homologue group changed depending on the GC/MS conditions such as the column; however, the reason for this is unclear.

Because CRM 7902-a and CRM 7904-a (PCB-fortified oils) are distributed with the base oil CRMs, CRM 7903-a and CRM 7905-a, users can adjust arbitrary concentration by mixing. Although densities of the CRMs were given as the information values, gravimetric mixing is recommended, because the oil samples, especially the insulation oil, are viscous. This allows one to validate analyses for PCB at the level of the Japanese criterion, and to validate PCB measurement with various instruments such as GC-ECD (LOD < $10 \mathrm{mg} \mathrm{kg}^{-1}$ ), GC/QMS (LOD $<1 \mathrm{mg} \mathrm{kg}^{-1}$ ), GC/HRMS (LOD $<0.1 \mathrm{mg} \mathrm{kg}^{-1}$ or ELISA (typical LOD $<1 \mathrm{mg} \mathrm{kg}^{-1}$ ). Thus, these CRMs are suitable for quality assurance of the sample analyses at the regulation level or lower.

\section{Conclusion}

The new CRMs, NMIJ CRM 7902-a, CRM 7903-a, CRM 7904-a, and CRM 7905-a, were issued by the NMIJ/AIST in March 2007, and they are now available together with their certificates from some distributors. The CRMs have reliable certified values of $11 \mathrm{PCB}$ congeners and are useful tools to validate the methods and skill for analysis of insulation oil and similar matrices such as waste oil, and provide a traceability link between routine methods and national standards.

In general, determination of PCBs is mainly based on measurements with ID-GC/HRMS equipped with a highresolution capillary column. This means that accuracies of risk assessments and pollution source identifications have been improved by congener-specific monitoring of the pollutants. At the same time, other instruments such as $\mathrm{GC} /$ QMS, GC-ECD, and ELISA measurement for the determination of total PCBs in wastes are also used for preliminary assessment. New NMIJ CRMs presented in this paper can be used for quality assurance at testing laboratories which analyze PCBs in waste using various instruments.

Acknowledgements The authors greatly appreciate the following cooperators. Collection of insulation oil was carried out at General Environmental Technos Co., Ltd. The base material (aminopropylsilica) for synthesis of the sulfoxide-bonded silica was kindly supplied by Dr. Kaneko, Sigma-Aldrich Japan.

\section{References}

1. Lang V (1992) J Chromatogr 595:1-43

2. Yao Y, Takasuga T, Masunaga S, Nakanishi J (2002) Chemosphere 46:1461-1469

3. JESCO homepage (2008) http://www.jesconet.co.jp/eg/index. html. Accessed 13 Feb 2008

4. Inagaki $\mathrm{K}$, Takatsu $\mathrm{A}$, Watanabe $\mathrm{T}$, Kuroiwa $\mathrm{T}$, Aoyagi $\mathrm{Y}$, Okamoto K (2004) Anal Bioanal Chem 378:1265-1270

5. Numata M, Yarita T, Takatsu A, Aoyagi Y, Matsuo M, Tsuda Y, Yamazaki M, Chiba K, Okamoto K (2007) Anal Bioanal Chem 378:1271-1276

6. Quinn TJ (1997) Metrologia 34:61-66

7. Ministry of Welfare, Japan (1992) Notification No. 192

8. Guitart R, Puig P, Gómez-Catalán J (1993) Chemosphere 27: $1451-1459$

9. Larsen B, Tilio R, Kapita S (1991) Chemosphere 23:1077-1084

10. Parris RM, Guenther FR, May WE, Chesler SN (1984) NBS Spec Publ 674:27-32

11. Tanaka N (2003) Kagaku Kogaku Ronbunshu 29:628-633 (in Japanese)

12. Matsubara H, Senba Y (2003) Kankyo Kagaku 13:1033-1040 (in Japanese)

13. Takada M, Toda H, Uchida R (2001) Chemosphere 43:455-459

14. Numata M, Aoyagi Y, Tsuda Y, Yarita T, Takatsu A (2007) Anal Chem 79:9211-9217

15. Chernetsova ES et al (2002) Mass Spectrom Rev 21:373-387

16. International Organization for Standardization (1993) Guide to the expression of uncertainty in measurement. ISO, Geneva

17. Linsinger TP, Pauwels J, van der Veen AMH, Schimmel $H$, Lamberty A (2001) Accred Qual Assur 6:20-25

18. International Organization for Standardization (1989) ISO guide 35: certification of reference materials - general and statistical principles. ISO, Geneva

19. Kitamura K, Takazawa Y, Hashimoto S, Choi JW, Ito H, Morita M (2004) Anal Chim Acta 512:27-37

20. Poster DL, Kucklick JR, Schantz MM, Porter BJ, Leigh SD, Wise SA (2003) Anal Biol Chem 375:223-241

21. Frame GM (1997) Fresenius J Anal Chem 357:701-713

22. Matsumura C, Tsurukawa M, Nakano T, Ezaki T, Ohashi M (2002) Kankyo Kagaku 12:855-865 (in Japanese)

23. Kim KS, Hirai Y, Kato M, Urano K, Masunaga S (2004) Chemosphere 55:539-553

24. Takasuga T, Senthil Kumar K, Matsumura T, Shiozaki K, Sakai S (2006) Chemosphere 62:469-484

25. NIST (1999) Certificate of analysis for SRM 3090: Aroclors in transformer oil. National Institute of Standards and Technology, Gaithersburg

26. BAM (1998) Reference material certificate: polychlorinated biphenyls in transformer oil, BAM CRM 5001. Federal Institute for Materials Research and Testing (BAM), Berlin

27. BCR (1991) Certificate of analysis for BCR CRM 420: chlorobiphenyls in waste mineral oil (low level). Community Bureau of Reference (BCR), Brussels

28. BCR (1993) Certificate of analysis for BCR CRM 449: chlorobiphenyls in waste mineral oil (high level). Community Bureau of Reference (BCR) Brussels

29. NIST (1999) Certificate of analysis for SRM 1581: polychlorinated biphenyls in oils. National Institute of Standards and Technology, Gaithersburg 